\title{
RTL implementation of image compression techniques in WSN
}

\author{
S. Aruna Deepthi ${ }^{1}$, E. Sreenivasa Rao ${ }^{2}$, M. N. Giri Prasad ${ }^{3}$ \\ ${ }^{1,3}$ Department of Electronics and Communication Engineering, Jawaharlal Nehru Technological University, India \\ ${ }^{2}$ Department of Electronics and communication Engineering, Vasavi college of Engineering, India
}

\begin{tabular}{l}
\hline \hline Article Info \\
\hline Article history: \\
Received Jul 10, 2018 \\
Revised Dec 17, 2018 \\
Accepted Dec 27, 2018 \\
\end{tabular}

Keywords:

NMF

PNMF

RTL

SVD

WSN

\begin{abstract}
The Wireless sensor networks have limitations regarding data redundancy, power and require high bandwidth when used for multimedia data. Image compression methods overcome these problems. Non-negative Matrix Factorization (NMF) method is useful in approximating high dimensional data where the data has non-negative components. Another method of the NMF called (PNMF) Projective Nonnegative Matrix Factorization is used for learning spatially localized visual patterns. Simulation results show the comparison between SVD, NMF, PNMF compression schemes. Compressed images are transmitted from base station to cluster head node and received from ordinary nodes. The station takes on the image restoration. Image quality, compression ratio, signal to noise ratio and energy consumption are the essential metrics measured for compression performance. In this paper, the compression methods are designed using Matlab. The parameters like PSNR, the total node energy consumption are calculated. RTL schematic of NMF SVD, PNMF methods is generated by using Verilog HDL.
\end{abstract}

Copyright (c) 2019 Institute of Advanced Engineering and Science. All rights reserved.

Corresponding Author:

S. Aruna Deepthi,

Department of Electronics and Communication Engineering,

Vasavi College of Engineering,

Hyderabad, India.

Email: sadeepthi@staff.vce.ac.in

\section{INTRODUCTION}

A sensor network [1], [2] consists of measuring, computing, and communication elements that give an administrator the ability to observe and react to events. While several sensors can be connected to controllers and processing stations directly (e.g., using local area networks), many sensors send the gathered data wirelessly to a centralized processing station. This is needed since many network applications require hundreds or thousands of sensor nodes, often set up in remote and unreachable areas. Figure 1 shows two sensor fields monitoring two different geographic regions and connecting to the Internet using their base stations [3].

The sensing and control technology comprise electric and magnetic field sensors; radio-wave frequency sensors; optical, electro-optic-, and infrared sensors; radars; lasers; location/navigation sensors; seismic and pressure-wave sensors; environmental parameter sensors (e.g., wind, humidity, heat); and biochemical national security-oriented sensors. Today's sensors can be described as "smart" inexpensive devices equipped with multiple onboard sensing elements; they are low-cost low-power multifunctional nodes that are logically homed to a central sink node [4].

Wireless Multimedia Sensor Networks (WMSNs) has broad applications [5] in industrial production, environmental monitoring [6]. The space-time relativity-based data compression algorithm mainly includes prediction coding and linear fitting method for time series. A prediction coding method is proposed by [7]. It evaluates the source data based on the time relativity of the source data. The WMSN's are different from traditional WSNs [8], [9] in case of data processing, and energy consumption of wireless transceiver. 


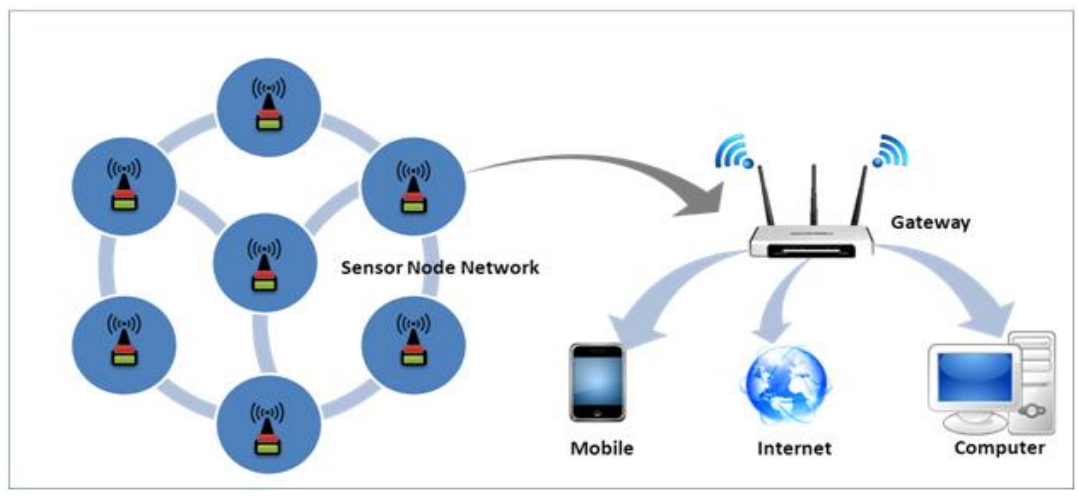

Figure 1. Wireless sensor networks

\section{PROPOSED METHOD}

In a WSN, the data transmission process [10] can be divided into data compression encoding and data decoding. The sending and receiving of images in WSNs can be shown in Figure 2. SVD, NMF, PNMF image compression methods are used.

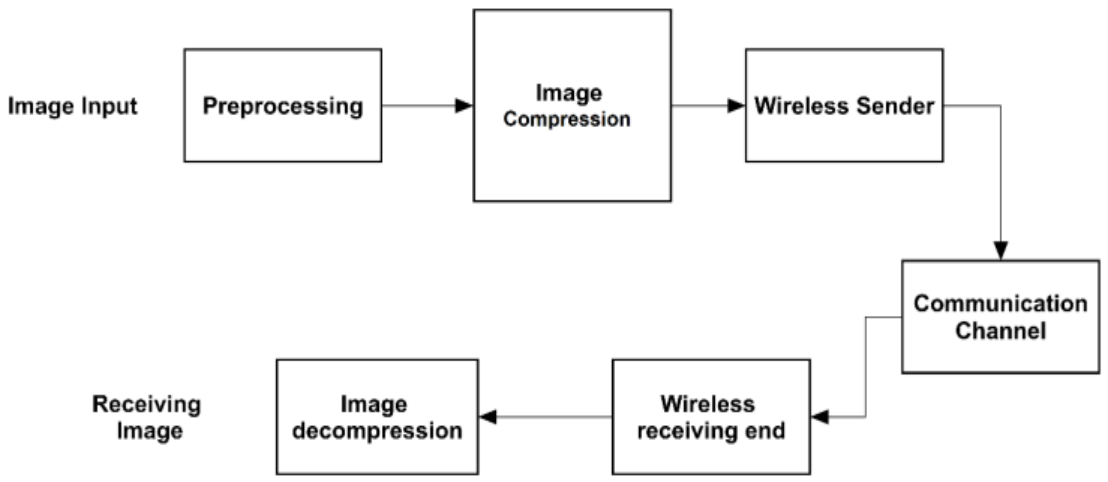

Figure 2. The image sending and receiving process

First, the given input image is resized to 512x512. The resized image is preprocessed and converted into corresponding pixel values using a Matlab program and stored in the memory file. The converted pixel values are input to image compression method which is developed by using Verilog HDL which is based on hardware which makes the process faster. Hardware/software co-simulation shown in Figure 3.

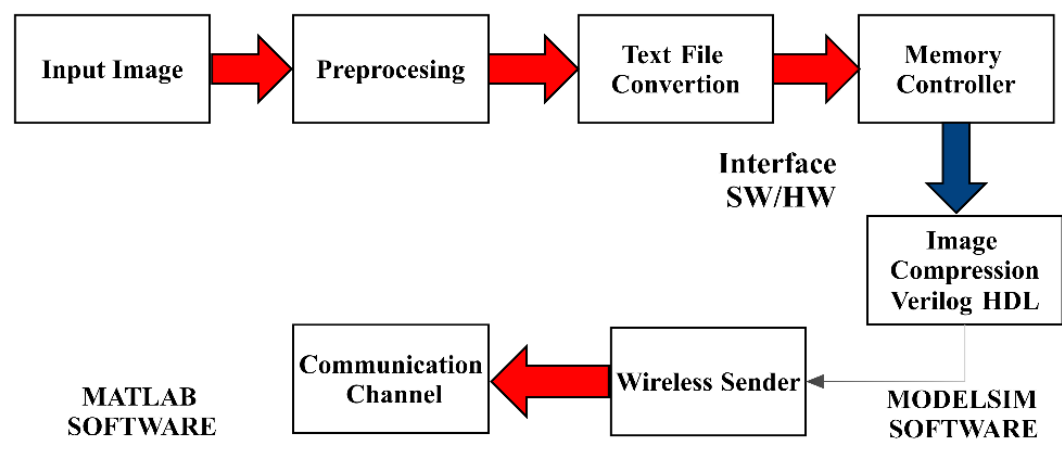

Figure 3. Hardware/software co-simulation 
Figure 4 shows the image values which are in files are stored in the memory of the ModelSim using the command "\$readmemh." for hexadecimal values and "\$readmemb" for binary values. The files contain hexadecimal values. HDLDAEMON command controls the server that supports interactions with HDL. Figure 5 show HDLDAEMON between MATLAB and ModelSim.

\begin{tabular}{|c|c|c|c|c|c|c|c|c|c|}
\hline 0001 & 0001 & 0001 & 0001 & 0001 & 0001 & 0002 & 0001 & 0000 & 0001 \\
\hline 0002 & 0002 & 0002 & 0001 & 0001 & 0001 & 0001 & 0002 & 0002 & 00 \\
\hline 0002 & 0002 & 0001 & 0002 & 0002 & 0002 & 0002 & 0002 & 0002 & 006 \\
\hline 0002 & 0002 & 0001 & 0002 & 0002 & 0001 & 0002 & 0002 & 0001 & 00 \\
\hline 0001 & 0002 & 0002 & 0001 & 0002 & 0002 & 0002 & 0002 & 0002 & $\theta$ \\
\hline 0002 & 0002 & 0003 & 0004 & 0003 & 0002 & 0002 & 0002 & 0003 & 000 \\
\hline 0002 & 0003 & 0003 & 0002 & 0003 & 0003 & 0005 & 0004 & 0002 & 00 \\
\hline$\theta 0 \mathrm{COF}$ & $00 \mathrm{D} 3$ & $\theta \theta D F$ & OOE3 & OOE2 & OOEO & $00 \mathrm{D} 7$ & $\theta \theta C B$ & $\theta 0 D C$ & $\theta 0 D$ \\
\hline $00 \mathrm{~B} 3$ & $\theta \theta C 4$ & $00 C 5$ & $00 C 4$ & $00 c 8$ & $\triangle O C E$ & $00 \mathrm{D} 6$ & $\theta \theta D A$ & $\theta \theta D B$ & 00 \\
\hline $00 D 9$ & 0007 & $00 D 1$ & $00 C 7$ & $\theta \theta c \theta$ & $00 C 2$ & $00 \mathrm{C} 1$ & Ө०BA & $\triangle O B C$ & 00 \\
\hline 0004 & 0003 & 0004 & 0003 & 0067 & $00 \mathrm{C} 8$ & OOCE & өөB० & OOA8 & $\theta 0$ \\
\hline $00 \mathrm{C} 6$ & $00 D 1$ & $\theta 0 C B$ & $00 C 2$ & OOB5 & $009 D$ & $00 A 8$ & $00 B 3$ & $00 A D$ & 00 \\
\hline $00 A 4$ & $008 \mathrm{~A}$ & $00 A 5$ & $00 A 5$ & $00 A 6$ & ООВ8 & $\theta \otimes C F$ & 0008 & OODD & $\theta 0$ \\
\hline OӨAE & $\theta \theta A D$ & $00 A 6$ & $009 \mathrm{C}$ & 0098 & $008 \mathrm{E}$ & 0088 & 0081 & $007 \mathrm{~A}$ & 00 \\
\hline 0002 & 0002 & 0002 & 0002 & 0002 & 0001 & 0001 & 0002 & 0002 & 00 \\
\hline 0003 & 0003 & 0003 & 0003 & 0003 & 0004 & 0004 & 0004 & 0005 & 00 \\
\hline 0005 & 0006 & 0006 & 0005 & 0006 & 0006 & 0007 & 0007 & 0004 & 00 \\
\hline 0008 & 0008 & 0009 & 0007 & 0006 & 0005 & 0004 & 0004 & 0005 & \\
\hline 0004 & 0002 & 0003 & 0004 & 0002 & 0002 & 0002 & 0003 & 0002 & 006 \\
\hline 0003 & 0003 & 0004 & 0003 & 0003 & 0003 & 0003 & 0002 & 0003 & \\
\hline
\end{tabular}

Figure 4. Pixel values in memory location

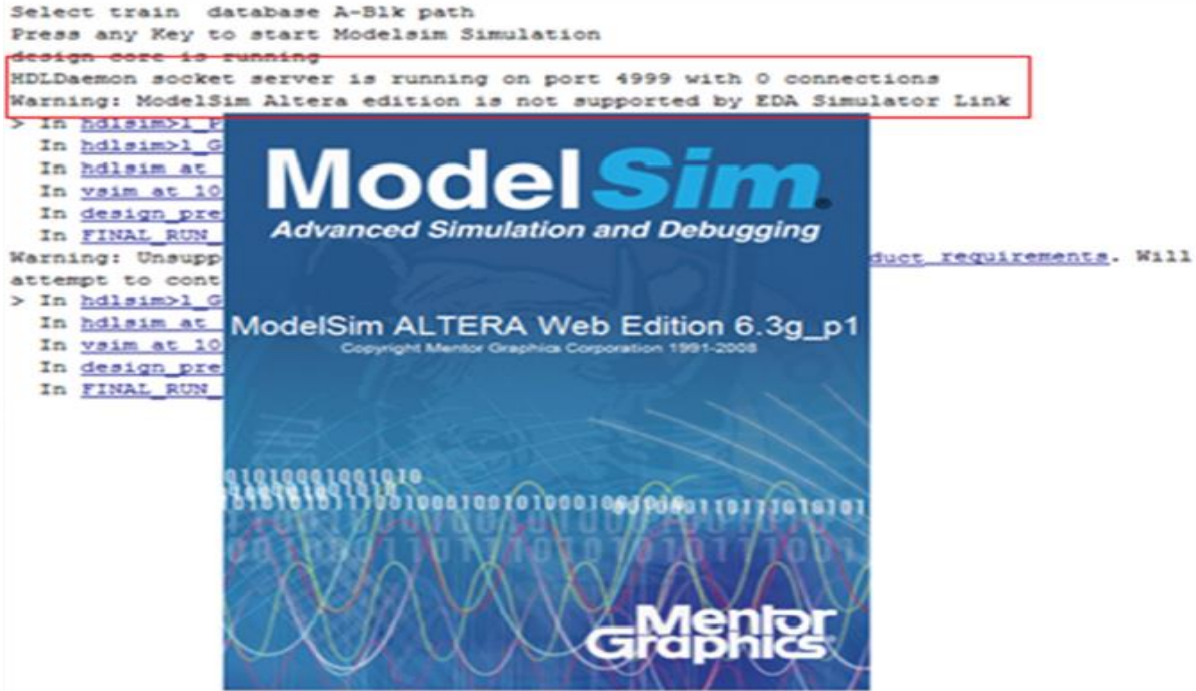

Figure 5. HDLDAEMON between MATLAB and ModelSim

\subsection{Image compression using NMF}

The NMF algorithm [11], [12] executes more iterations to get better image quality. Images are divided into several blocks, and adaptive image compression algorithm is taken based on the NMF [13], [14] to process each image block. The compression ratio of the NMF [15], [16] method depends on the size of the rank of the base matrix. Therefore, the compression ratio can be modified with the demands in the energy consumption. Lower quality is selected to improve the compression ratio to save energy. Suppose an original image with the size of $\mathrm{m} \times \mathrm{n}$, is divided into several blocks the dimensions of which are $\mathrm{p} \times \mathrm{q}$. The image compression ratio is given by (1).

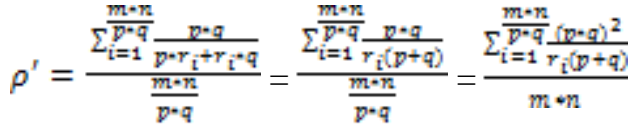




\subsection{Image compression using SVD}

Singular Value Decomposition (SVD) is represented by the (2).

$$
\mathrm{G}=\mathrm{LD} R^{T}
$$

where $\mathrm{G}$ is a $\mathrm{m} \times \mathrm{n}$ matrix

Image compression [17] involves reducing the redundancies in an image which are in the form of

1. Psycho-visual redundancy, which is due to the limitations [18] of the human visual system to interpret fine details in an image. (i.e., visually non-essential features)

2. Interpixel redundancy,

3. Coding redundancy

Removing the redundancies is [19],[20] reducing the number of bits in an image without the decrease in the image quality. However, merely applying SVD [21], [22] on an image do not compress it and a few singular values were retained while other unique values were ignored. The singular values were taken in descending order on the diagonal of $\mathrm{D}$, and that first singular value contains the most significant amount of information, and following singular values contain decreasing amounts of image information. Thus, the lower singular values containing less information can be ignored without distortion in the image.

From the property 1 of SVD, it can be referred that "the rank of G is taken as the number of nonzero singular values". But if the lower order singular values after the rank of the matrix have negligible values, they are considered as noise.

In (2) above can also be written as

$$
G=1_{1} \sigma_{1} r_{1}^{T}+1_{2} \sigma_{2} r_{2}^{T} \ldots \ldots \ldots \ldots+1_{y} \sigma_{y} r_{y}^{T} \ldots \ldots \ldots+1_{\mathbb{N}} \sigma_{\mathbb{N}} r_{\mathbb{N}}^{T}
$$

where $r$ is the rank of $\mathrm{G}$.

From property 1 of SVD (refer section 3 above), it follows that truncating (3) till $r$ values does not make any significant change in the image. But compression will be very less while the image quality is same.For good amount of compression to be achieved, only the first k values of (4)

$$
G=1_{1} \sigma_{1} r_{1}^{T}+1_{2} \sigma_{2} r_{2}^{T} \ldots \ldots \ldots+1_{k} \sigma_{k} n_{k}^{T}
$$

where $\mathrm{k}<\mathrm{r}$

The image reconstructed will reduce[2] the storage space requirement to $\mathrm{k}^{*}(\mathrm{~m}+\mathrm{n}+1)$ bytes as against the storage space requirement of $m^{*} \mathrm{n}$ bytes of the original uncompressed image. Now, compression is achieved if the storage space required by the compressed image is less than that required by the original image.

$$
\mathrm{m} * \mathrm{n}>\mathrm{k} *(\mathrm{~m}+\mathrm{n}+1)
$$

\subsection{Image compression using PNMF}

In NMF, $\mathrm{W}$ and $\mathrm{H}$ has $\mathrm{r} \times(\mathrm{m}+\mathrm{n})$ free parameters[20]. Consider $\mathrm{V}$ and $\mathrm{W}$ are column vectors and $\mathrm{H}$ is a scalar: obviously, there are many solutions $\mathrm{W}=1 / \mathrm{H} \mathrm{V}$ with $\mathrm{H}$ arbitrary. Based on this, a novel method called Projective Non-negative Matrix Factorization (PNMF) is taken as the solution to the problem

$$
\min _{W \geq 0}\left\|V-V W^{T} V\right\|
$$

where $\|\cdot\|$ is a matrix norm.

The most useful norm is the Euclidean distance between two matrices A and B, or the Frobenius matrix norm of their difference:

$$
\|A-B\|^{2}=\sum_{i, j}\left(A_{i j}-B_{i j}\right)^{2}
$$

a divergence of matrix A from B defined as

$$
D(A \| B)=\sum_{i, j}\left(A_{i j} \log \frac{A_{i j}}{B i j}-A_{i j}+B_{i j}\right)
$$

Both Euclidean distance and divergence are lower bounded by zero, and vanish if and only if $\mathrm{A}=\mathrm{B}$. The PNMF method seems to offer some advantages as compared to NMF. The first one has increased orthogonality of the basis vectors. This is due to the similarity of the criterion [23] to SVD. Removing the positivity constraint but keeping the rank constraint, an orthogonal eigenvector basis is a solution. 
For positive bases, orthogonality is related to sparseness. Consider the case in which the $\mathrm{V}$ matrix is a training set, and the goal is to find the representation not only for the columns of $\mathrm{V}$ but for new vectors, too. For PNMF, the representation for any column of V, say v, is simply WWT v and that can be easily computed for a new vector, too. In NMF, there is no such natural representation because both $\mathrm{W}$ and $\mathrm{H}$ are needed, and matrix $\mathrm{H}$ has only n columns. The extra column in $\mathrm{H}$ would have to be recomputed from the criterion. Third, as pointed out by [24]. NMF has a close relation to clustering.

\section{RESULTS}

\subsection{Relative parameters in simulation experiment}

In this paper Matlab is used for compressing images using NMF, SVD and Verilog is used for generating RTL schematic of SVD and NMF algorithms in WSN. Figure 6 shows input image of size $512 \times 512 \times 8$-bit grayscale images is collected by camera nodes periodically. Here 15 cameras cover each detection range of a 100x100 m rectangular area, and their sensing radius is $11 \mathrm{~m}$. Eleven ordinary nodes are deployed in the communication range of each camera node. The simulated network structure for three methods is shown in Figure 7.

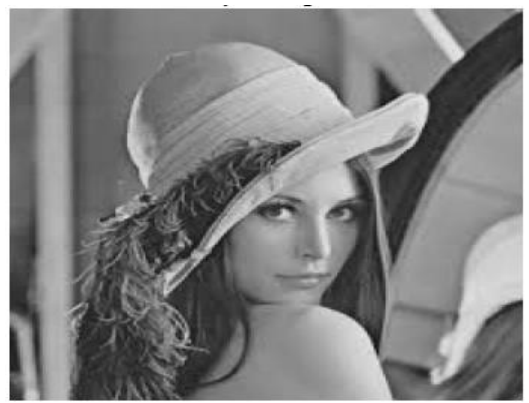

Figure 6. Input image 512x512

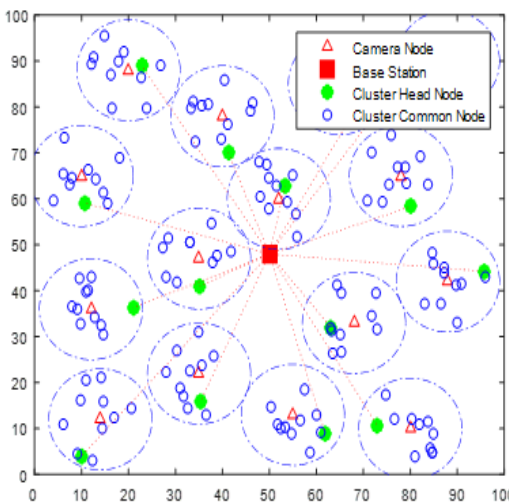

(a)

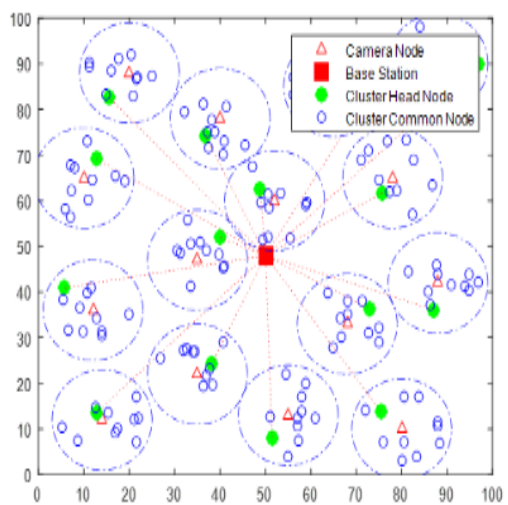

(b)

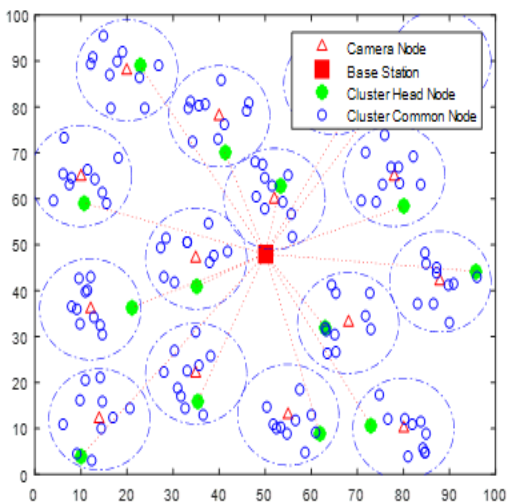

(c)

Figure 7 (a). Network structure of NMF compression mechanism (b) Network structure of PNMF compression mechanism (c) Network structure of SVD compression mechanism

\subsection{Analysis of energy consumption}

Figure. 8 shows NMF energy distribution figure when sending a $512 \times 512 \times 8$-bit image to the base station. The simulation result indicates that the use of the three compression methods described in this paper reduces the average energy consumption of the camera nodes by nearly an order of magnitude lower than the centralized approach. The energy consumption of nodes has a stable balance, which is helpful to improve the lifetime of network and extend the life cycle. In the Table 1, PSNR values expressed in $\mathrm{dB}$ are used as a measure of image quality. Table 2 show xilinx ISE device utilization factor for NMF, SVD, PNMF methods. 


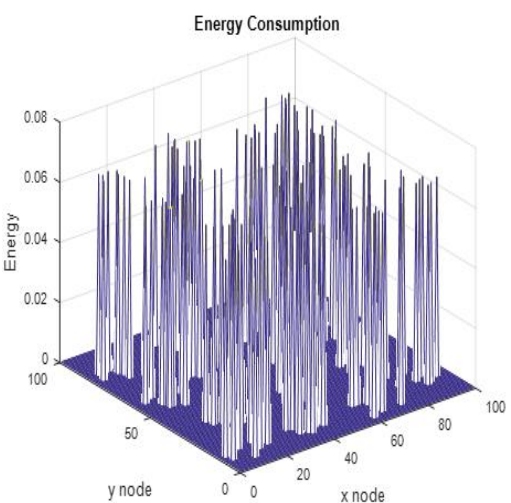

(a)

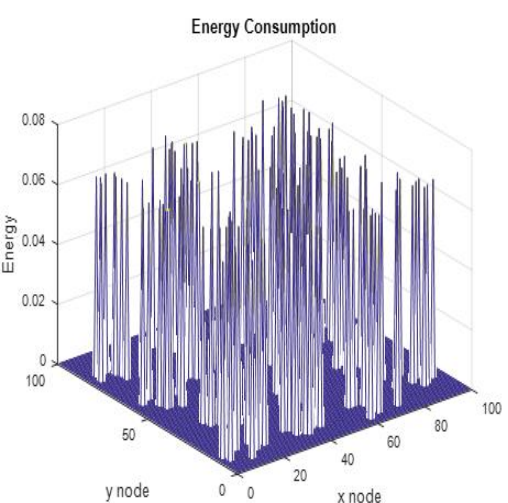

(b)

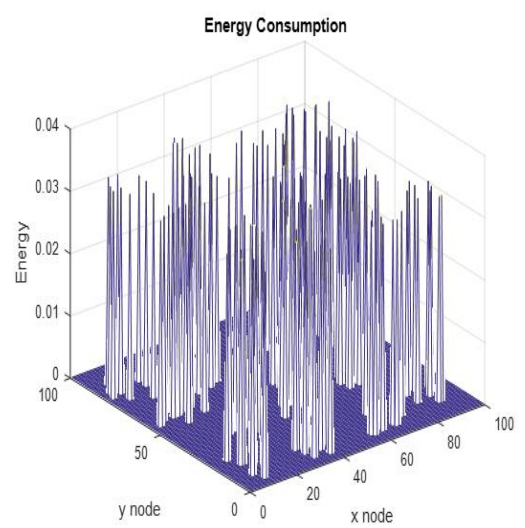

(c)

Figure 8 (a). Energy distribution of NMF image compression network (b) Energy distribution of PNMF (c) Energy distribution of SVD image compression network

Table 1. PSNR Value for Three Compression

Methods with the Image Input size of $512 \times 512$

\begin{tabular}{cc}
\hline Compression Method & PSNR Value \\
\hline SVD & 12.0048 \\
NMF & 17.0048 \\
PNMF & 20.1720 \\
\hline
\end{tabular}

Table 2. Xilinx ISE Device Utilization Factor for NMF, SVD, PNMF Methods

\begin{tabular}{lc}
\hline \multicolumn{1}{c}{ Logic Utilization } & NMF, PNMF, SVD \\
\hline Number of Slices & 1817 \\
Number of Slices in Flip Flops & 1776 \\
Number of 4 input LUTs & 3356 \\
Number of bonded IOBs & 77 \\
Number of GCLKs & 1 \\
Maximum Frequency & $80.512 \mathrm{MHz}$ \\
\hline
\end{tabular}

The design is based block splitting concept with block size of $8 \times 8$ the device utilization factor will be similar to all the three methods. Xilinx ISE 14.1 version with family Virtex 6-XC6VLX757 and package FF784 is used. Figure 9 show RTL schematic of NMF, SVD, PNMF methods.
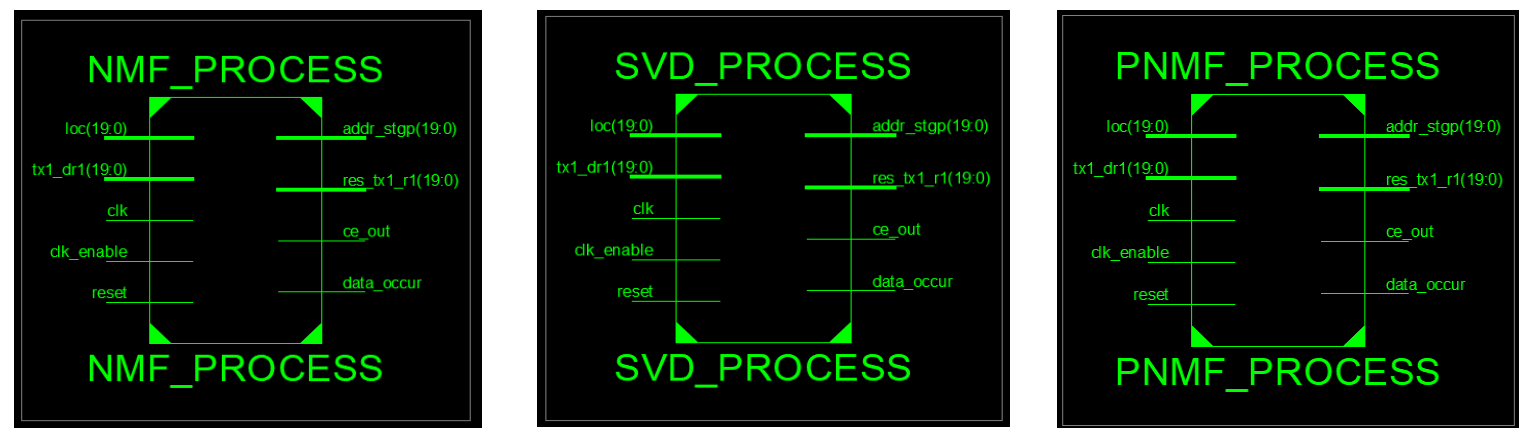

Figure 9. RTL schematic of NMF, SVD, PNMF methods

\section{CONCLUSION}

In this paper, the different image compression methods like SVD, NMF, and PNMF in WSNs are designed. An attempt is made to connect Matlab and Verilog HDL.Images are compressed and transferred using Matlab and RTL schematic is generated using Verilog HDL. Image quality, compression ratio, signal to noise ratio and energy consumption are the most vital metrics calculated using Matlab. Simulation results show that the PNMF-based image compression mechanism provided better PSNR and SVD based image compression provides alleviate the energy consumption of camera nodes which are the key roles in the network. 


\section{REFERENCES}

[1] Abdullah AB, Ragab. K\& zaman. N, "Wireless Sensor Networks and Energy Efficiency," published by IGI Global, pp 1-18, 2012.

[2] Ahonen.T, Pietikainen, M.Zhao, G\&Matas.J,. "Rotation-invariant image and video description with local binary pattern feartures," IEEE Transactions on Image Processing, 1465-1477, 21(4), 2012.

[3] Bengio.Y.Courville. A.C \& Vincent.P., "Unsupervised feature learning and deep learning: A Review and new Perspectives," Departmant of computer science and operation research, University of Montreal, Montreal Canada

[4] Brunello.D, Calvagno, G.Mian, G.A\&Rinaldo.R, "Lossless compression of video using temporal information," IEEE Transactions on Image Processing, pp. 132-139, 2003.

[5] Chang.N.B, Liu.M, "Optimal competitive algorithms for opportunistic spectrum access, ” IEEE Journal on selected areas in communications, pp. 1183-1192, 2008.

[6] Cheng, C, Chen. X, Wang.S \& Yang.Y, “Amultilayer Improved RBM network based image compression method in Wireless sensor networks," 2016.

[7] Dargie.W \& Poellabauer.C, "Fundamentals of Wireless sensor Networks: theoty and Practice," John Wiley and Sons Ltd.West Sussex, UK:doi:10.1002/9780470666388.

[8] Ding.C.He, X \& Simon H, "On the equivqlence of Nonnegative Matrix factorization and Spectral clustering," Proceedings of Conference data mining, pp. 606-610, 2005.

[9] Dipayan, "Deep learning with Hadoop," Published by Packt Publishing, 2017.

[10] Gao.Y, Li, W.X, L.Sun.G.L, \&Zhang.C "Reserch on data compression algorithm based on prediction coding for wireless sensor nodes," Proceedings of international forum on information technology and applications (IFITA-09) vol. 1, pp. 283-286.

[11] Huang.K.Sidiropoulos, N \& Swami, "A non negative matrix factorization revisited: Uniqueness and algorithm for symmetric decoposition,” IEEE transactions on signal processing, pp. 211-224, 2014.

[12] Priyanka Mekala;Jeffrey Fan;Wen Cheng Lai and Ching Wen Hsue, "Gesture recognition using neural networks based on HW/SW cosimulation platform," Hindawi journal Advances in software engineering, 2013 http://dx.doi.org/10.1155/2013/707248.

[13] Kahu.S. \& Rahate.R, "Image compression using SVD," International journal of advancements in research and technology, No. 8, pp. 244-248, 2013.

[14] Kim.J \& Kyung.C.M. "A lossless embedded compression using significant bit truncation for HD video coding," IEEE transactions on systems for video technology, pp. 848-860, 2010.

[15] Tao Li. \& Chris Ding, "The Relationships among various Nonnegative Matrix factorization," Sixth international conference on Data mining," http://doi.org/10.1109/ICDM.2006.160, 2006.

[16] Li.H, Li.Z \& Wen. C, "Fast mode decision algorithm for interframe coding in fully scalable video coding," IEEE transactions on circuits and systems for video technology, PP889-898

[17] Ma.Z.Wang.W, Xu.M \& Yu.H "Advanced screen content coding using color table and index map" IEEE transactions on image processing, pp. 4399-4412.

[18] Minoli.D, Sohraby. K, Znati.T, "Wireless sensor Networks, Technology, Protocols and applications," John Wiley \& Sons, Inc Hoboken, $\mathrm{Nj}$ :doi:10.1002/047011276X.

[19] Swami.A, Win Hong, Zhao. Q, "Wireless Sensor Networks signal processing and communications perspectives," John Wiley \& Sons Ltd.West Sussex, UK.

[20] Wang.K.J\&Zuo .C.T, "Improvements of non negative matrix factorization for image extraction," Appl.Res.Comput.

[21] Xiang Yu.L, Xiao-Chun.Y \& Ya-Zhe.W, "Facing the wireless sensor networks streaming data compression technology," Computer Science vol. 34, no. 2, pp. 141-143, 2007.

[22] Baghouri Mostafa, Chakkor saad, Hajraoui Abderrahmane, "Firefly algorithm to improve threshold distributed energy efficient clustering algorithm for heterogenous Wireless sensor Networks," IAES International Journal of Artificial Intelligence, vol. 6, No. 3, pp. 91-99, 2017.

[23] K.Panimozhi, G.Mahadevan, "QOS Frame work for a multi stack based heterogenous Wireless sensor network," International journal of Electrical and Computer Engineering (IJECE ), vol. 7, no. 5p, pp. 2713-2720, 2017.

[24] Emy Setyaningsih, Agus Harjoko "Survey of Hybrid Image compression Techniques," International Journal of Electrical and Computer Engineering (IJECE), vol. 7, No. 4, pp. 2206-2214, 2017. 